\title{
Carbon monoxide releasing molecule- 2 attenuated ischemia/reperfusion-induced apoptosis in cardiomyocytes via a mitochondrial pathway
}

\author{
SHEN ZHAO ${ }^{1-3}$, QINGMING LIN ${ }^{1-3}$, HENG LI $^{1,2,4}$, YUMIN HE ${ }^{1,2}$, XIANGSHAO FANG ${ }^{1,2}$, \\ FENG CHEN $^{3}, \mathrm{CHANGWEI} \mathrm{CHEN}^{4}$ and ZITONG HUANG ${ }^{1,2}$
}

\begin{abstract}
${ }^{1}$ Department of Emergency, Sun Yat-sen Memorial Hospital; ${ }^{2}$ Institute of Cardiopulmonary Cerebral Resuscitation, Sun Yat-sen University, Guangzhou, Guangdong 510120; ${ }^{3}$ Provincial Clinical Medical College of Fujian Medical University, Fuzhou, Fujian 350001; ${ }^{4}$ Taiping People's Hospital of Dongguan City, Dongguan, Guangdong 523900, P.R. China
\end{abstract}

Received June 2, 2013; Accepted November 27, 2013

DOI: $10.3892 / \mathrm{mmr} .2013 .1861$

\begin{abstract}
Carbon monoxide (CO) is an endogenous gaseous transmitter that exerts multi-protection in ischemia/reperfusion $(\mathrm{I} / \mathrm{R})$ injury, but few experimental studies regarding $\mathrm{CO}$ on myocardial I/R-induced apoptosis, as well as its underlying mechanism have been conducted. The present study was designed to investigate whether $\mathrm{CO}$ released from CO-releasing molecule-2 (CORM-2) is capable of ameliorating myocardial I/R-induced apoptosis via a mitochondrial apoptotic pathway. Primary cultures of neonatal rat cardiomyocytes were randomly distributed into four groups: Control, $\mathrm{I} / \mathrm{R}$ (cultured cardiomyocytes were subjected to $2 \mathrm{~h}$ simulated ischemia followed by $4 \mathrm{~h}$ reperfusion), CORM-2 and inactive CORM-2 (iCORM-2) groups (20 $\mu \mathrm{M}$ CORM-2 and $20 \mu \mathrm{M}$ iCORM-2 were administered at the beginning of reperfusion following ischemia, respectively). Flow cytometric analysis showed that CORM-2 treatment significantly decreased apoptosis of cardiomyocytes triggered by simulated I/R . CORM-2 partially recovered mitochondrial respiration and ultrastructure alteration, and lowered caspase- 3 expression and the release of cytochrome $c$. Furthermore, CORM-2 partly reduced $\mathrm{BAK} / \mathrm{BAX}$ expression in mitochondria, as well as the BAX level in the cytoplasm. Cardioprotection is lost when CORM-2 is replaced by iCORM-2. CORM-2 treatment, at the time of reperfusion, was concluded to attenuate myocardial I/R-induced apoptosis. The protection mechanisms may be targeted to the mitochondria and involved in the inhibition of the BAK/BAX-mediated intrinsic pathway.
\end{abstract}

Correspondence to: Professor Zitong Huang, Department of Emergency, Sun Yat-sen Memorial Hospital, Sun Yat-sen University, 107 Yanjiangxi Road, Guangzhou, Guangdong 510120, P.R. China E-mail: huangzitong_jzk@163.com

Key words: apoptosis, carbon monoxide, mitochondrion, ischemia/reperfusion, cardiomyocytes

\section{Introduction}

Myocardial reperfusion injury following transient focal or systemic ischemia is a complex pathophysiological process that induces extensive cell death, and a marked portion of which initiate mitochondrial-mediated apoptosis $(1,2)$. A previous study has implicated the mitochondrial ultrastructure and respiratory function involved in myocardial contractility failure in stimulated ischemia-reperfusion (I/R) (3). However, the mechanisms of molecular signaling pathways and cytoprotective strategies targeting mitochondria during I/R-induced apoptosis require further investigation.

To ameliorate the severity of cardiac $\mathrm{I} / \mathrm{R}$, a number of anti-apoptotic therapies targeting mitochondria are currently being investigated, including pharmacological and genetic inhibition (4). Cardiac myocytes express various members of the BCL-2 family, including anti-apoptotic and pro-apoptotic BCL-2 proteins, which control a critical checkpoint in the mitochondria by preventing or promoting apoptosis $(5,6)$. The anti-apoptotic factors include BCL-2, BCL- $\mathrm{X}_{\mathrm{L}}$ and MCL-1. The pro-apoptotic proteins are further divided into the multi-domain members BAK and BAX, in addition to a diverse group of 'BH3-only' molecules. BAK and BAX are required for I/R-induced cell death by the mitochondrial or intrinsic pathway (7). In viable cells, monomeric BAK is constitutively localized at the mitochondrial outer membrane (MOM), whereas inactive BAX are located in the cytosol. Upon stress, active homo-oligomerized BAK or BAX mediated membrane permeability and the efflux of cytochrome $c$ from mitochondria, which led to the activation of caspases (8). Mouse embryonic fibroblasts (MEFs) with genetic depletion of BAK or BAX were less sensitive to truncated BID (tBID)-induced MOM permeabilization and apoptosis, which is due to the reduced recruitment of pro-apoptotic BCL-2 family proteins to MOM (9).

In the view of the mounting evidence supporting a homeostatic and cytoprotective role of carbon monoxide (CO) in biological systems, much interest has focused on harnessing the actions of this molecule for therapeutic purpose (10). Stimulation of endogenously generated CO or low dose of 
exogenous $\mathrm{CO}$ targeting mitochondria has shown activity, including oxidative phosphorylation and cell death inhibition (11). CO treatment enhanced cytochrome $c$ oxidase (COX) activity and BCL-2 expression as well as their interaction, which protects astrocytes against oxidative stress-induced apoptosis by improving mitochondrial oxidative phosphorylation (12). In an oxidative stress model by Conde de la Rosa et al (13), CO protected primary hepatocytes against superoxide-anion-induced apoptosis. To the best of our knowledge, there is little information regarding the effect of $\mathrm{CO}$ on pro-apoptotic BCL-2 protein expression during myocardial I/R injury. Furthermore, it has been demonstrated that CO-releasing molecules (CORMs) also deliver $\mathrm{CO}$ and these compounds have since emerged as potential therapeutic agents for the treatment of various cardiovascular disorders $(10,14)$. Notably, CORMs treatment is associated with low or minimal formation of carboxyhemoglobin and is therefore considered a safer alternative to CO gas inhalation (15). Recent studies reported that the tricarbonyldichlororuthenium (II) dimer (CORM-2) may attenuate small bowel and kidney damage caused by I/R $(16,17)$.

Based on the above information, CORM-2 was hypothesized to ameliorate myocardial apoptosis triggered by $\mathrm{I} / \mathrm{R}$ through the mitochondrial pathway. In the current study, myocardial I/R injury was induced in neonatal rat cardiomyocytes and the effects of CORM-2 on mitochondrial respiratory function were investigated, as well as the expression of MOM permeabilization-related pro-apoptotic protein, BAK/BAX in cellular extracts.

\section{Materials and methods}

Ethics statement. The study was approved by the Animals Ethics Committee of Sun Yat-sen University (Guangzhou, China) and all animals received humane care in compliance with the Guide for the Care and Use of Laboratory Animals published by the National Institute of Health (NIH publication 86-23, revised 1986).

Reagents. Tricarbonyldichlororuthenium (II) dimer (CORM-2) was purchased from Sigma-Aldrich (St. Louis, MO, USA); cellular mitochondria isolation kit from Beyotime Inc. (Jiangsu, China), respiratory control ratio (RCR) quantitative testing kit from Genmed Scientifics Inc. (Wilmington, DE, USA), anti-BAK and anti-BAX antibodies from Abcam (Cambridge, UK) and anti-caspase-3 and anti-cytochrome $c$ antibodies from Santa Cruz Biotechnology, Inc. (Heidelberg, Germany).

Cell cultures and treatment. In brief, single cells were dissociated from minced hearts $\left(1 \mathrm{~mm}^{3}\right)$ of Sprague-Dawley (SD) rats (age, $1-3$ days) with $0.125 \%$ trypsin (fresh preparation; Gibco-BRL, Carlsbad, CA, USA) and $0.05 \%$ type I collagenase (fresh preparation; MP Biomedicals, Santa Ana, CA, USA). Cell solution was filtered with $100 \mu \mathrm{m}$ nylon mesh and suspended in low-sugar Dulbecco's modified Eagle's medium (DMEM) containing 10\% (v/v) fetal bovine serum (both Gibco-BRL) and 1\% (v/v) penicillin-streptomycin solution (Mediatech, Inc., Manassas, VA, USA) and pre-plated for $60 \mathrm{~min}$ to reduced contamination of non-myocytes. The non-adherent cardiomyocytes were separated and cultured at a density of $2 \times 10^{5}$ cells $/ \mathrm{cm}^{2}$ with the aforementioned medium supplemented with $0.1 \mathrm{mM}$ 5'-bromo-2'-deoxyuridine (BrdU; Sigma-Aldrich, St. Louis, MO, USA) in a $37^{\circ} \mathrm{C} / 5 \% \mathrm{CO}_{2}$ humidified incubator (Mini Galaxy A, RS Biotech Inc., Irvine, UK). After a three-day cultivation, cardiac myocytes were randomly divided into four groups: i) Control: Cells were continuously cultured for $6 \mathrm{~h}$ in the cellular-cultured medium; ii) I/R: Cells were subjected to $2 \mathrm{~h}$ simulated ischemia followed by $4 \mathrm{~h}$ of reperfusion; iii) CORM-2: $20 \mu \mathrm{M}$ CORM-2 in $0.2 \%$ dimethylsulfoxide (DMSO) was administered to the culture medium at the beginning of reperfusion and iv) inactive CORM-2 (iCORM-2): $20 \mu \mathrm{M}$ iCORM-2 (CO depleted) was added as described previously. iCORM-2 was produced by preparing CORM-2 in DMSO and leaving it in a sealed, sterile container exposed to light for $48 \mathrm{~h}$ and bubbling $\mathrm{N}_{2}$ gas to remove the residual solubilized CO. For controls, $0.2 \%(\mathrm{v} / \mathrm{v})$ DMEM was added to the medium of the first two groups at the beginning of reperfusion and equivalent volumes of medium in each group was added. Only cultures consisting of $>95 \%$ actin-positive cells, determined by counting 300 cells in three fields, were subjected to analysis.

Simulated $I / R$. In this study, hypoxia in ischemic buffer solution (serum- and glucose-free DMEM pre-gassed with $95 \% \mathrm{~N}_{2}$ and $5 \% \mathrm{CO}_{2}$ for $\geq 5 \mathrm{~min}$ ) was utilized to mimic the ischemic process in vivo, while oxygen, as well as serum and glucose restoration stood for reperfusion. The medium was replaced with the ischemic buffer solution, the cells were placed in a hypoxia chamber filled with $5 \% \mathrm{CO}_{2}$ and $0.3 \% \mathrm{O}_{2}$ at $37^{\circ} \mathrm{C}$ for $2 \mathrm{~h}$. Following hypoxia exposure, the cells were reoxygenated with $5 \% \mathrm{CO}_{2}$ and $19 \% \mathrm{O}_{2}$ at $37^{\circ} \mathrm{C}$ for a further $4 \mathrm{~h}$ in normal cellular-cultured medium.

Cell viability assay. Cell viability was determined based on methyl thiazolyl tetrazolium (MTT) metabolism (18). The myocytes were seeded in a 96-well plates at a density of $1 \times 10^{5}$ cells/well and incubated with $5 \mathrm{~g} / 1 \mathrm{MTT}$ for $4 \mathrm{~h}$ at $37^{\circ} \mathrm{C}$. The reaction was stopped by the addition of $150 \mu \mathrm{l}$ diphenylamine solution and the absorbance of the blue formazan derivative was read at $570 \mathrm{~nm}$ using a spectrophotometer. The percentage of cell viability was calculated by the following formula: $\%$ cell viability $=$ (mean absorbance in test well)/(mean absorbance in control well) x 100 .

Lactate dehydrogenase $(L D H)$ activity assay. The extent of cellular injury was monitored by spectrophotometrically measuring LDH activity in the culture medium with an LDH activity assay kit (Sigma-Aldrich).

Preparation of mitochondria. The mitochondrial isolation procedures were completed within $1 \mathrm{~h}$ of cell collection. Myocardial mitochondria were extracted by deferential centrifugation in mitochondria extraction buffer at $4^{\circ} \mathrm{C}$, as described previously (3). With a $0.25 \%$ solution of crude trypsin, adherent cells were digested and centrifuged (500 x g). The cell pellets were mixed with mitochondria extraction buffer and placed in a glass homogenizer for homogenizing. Following differential centrifugation $(600 \mathrm{x} \mathrm{g}$ for $10 \mathrm{~min}$ followed by $11,000 \mathrm{x} \mathrm{g}$ for $10 \mathrm{~min}$ ), final deposits 
were stored within mitochondrial stocks and the supernatants were cytosol exclusive of mitochondria.

Mitochondrial respiration. Mitochondrial respiratory function was measured at $25^{\circ} \mathrm{C}$ using an Oxygen Electrode (Oxygrph ${ }^{\mathrm{TM}}$, Hansatech Instruments, King's Lynn, UK). Real-time changes of oxygen concentration were recorded via Oxygraph ${ }^{\mathrm{TM}}$ software (Hansatech Instruments). According to the manufacturer's instructions, calibration, followed by zero-oxygen line establishment with sodium hydrosulfite $\left(\mathrm{NaO}_{2} \mathrm{SSO}_{2} \mathrm{Na}\right)$ and sensibility test was completed in the chamber prior to measurement. While testing, $2.5 \mathrm{ml}$ respiration medium: $225 \mathrm{mM}$ mannitol, $70 \mathrm{mM}$ sucrose, $1 \mathrm{mM}$ EDTA, $10 \mathrm{mM}$ potassium phosphate, $0.1 \%$ bovine serum albumin ( $\mathrm{pH} 7.4)$ was initially agitated with a magnetic stirrer (Hansatech Instruments) in the sealed chamber. The medium was saturated with oxygen to reach an oxygenic concentration of $\sim 280 \mathrm{nmol} / \mathrm{ml}$ and mitochondrial suspension $(20 \mu \mathrm{l})$ was added to the medium. The rate of state IV respiration is presented as a gently declined curve and was initiated by adding $20 \mu \mathrm{l}$ disodium succinate. The rate of state III respiration was further initiated by the addition of $20 \mu \mathrm{l}$ adenosine 5'-diphosphate (ADP). Real-time oxygen concentration was recorded for the average oxygen consumption rate. An abrupt drop in oxygen concentration indicated fast phosphorylation of ADP added to adenosine 5'-triphosphate (ATP). The RCR, an index of integrality of membranes and the coupling of oxidative phosphorylation in mitochondria, was calculated as the ratio of the respiratory rate in state III to that in state IV.

Annexin V-fluorescein isothiocyanate (FITC)/propidium iodide (PI) double marking flow cytometry. According to the manufacturer's instructions, cells were rinsed three times with ice-cold PBS and stained with Annexin V-FITC (Calbiochem-Merck Co., Darmstadt, Germany) for $15 \mathrm{~min}$ at room temperature in $200 \mu \mathrm{l}$ binding buffer. Subsequent to this, $300 \mu 1$ binding buffer was added, the cells were stained with propidium iodide (PI) for $30 \mathrm{~min}$ at $4^{\circ} \mathrm{C}$ and their fluorescence was analyzed by flow cytometery (Beckman Coulter, Brea, CA, USA). The percentage of apoptotic cells was determined using WinMDI version 2.9 (Scripps Research Institute, San Diego, CA, USA).

Transmission electron microscopy. Ventricular cells were fixed by paraformaldehyde and glutaraldehyde (2:1 in volume), dehydrated in acetone series and embedded in epon 812 . Ultrathin sections $(50-70 \mathrm{~nm})$ were sliced and stained with uranyl acetate and lead acetate and examined under a transmission electron microscope (Tecnai G2 Spirit Twin; FEI, Hillsboro, OR, USA).

Detection of caspase-3 activation. Total proteins (20 $\mu \mathrm{g}$ per lane) were separated by $10 \%$ SDS-PAGE and electrophoretically transferred onto polyvinylidene fluoride membranes. The membranes were blocked with Tris-buffered saline containing $5 \%$ non-fat milk for $2 \mathrm{~h}$ at room temperature and incubated with rabbit anti-caspase-3 monoclonal antibody (1:500) overnight at $4^{\circ} \mathrm{C}$. The membranes were washed three times in 1X Tris-buffer saline with Tween-20 (TBST) and incubated with anti-IgG antibody conjugated with alkaline phosphatase,
Table I. Effects of CORM-2 on cell viability and LDH activity in cardiomyocytes subjected to I/R injury.

\begin{tabular}{lcc}
\hline Groups & MTT (optical density, \%) & LDH $\left(\mathrm{U} / 1^{-1}\right)$ \\
\hline Control & $92.6 \pm 7.3^{\mathrm{a}}$ & $18.6 \pm 1.3$. \\
I/R & $56.3 \pm 8.8^{\mathrm{b}}$ & $55.4 \pm 1.8^{\mathrm{b}}$ \\
CORM-2 & $70.3 \pm 9.5^{\mathrm{a}}$ & $30.3 \pm 2.5^{\mathrm{a}}$ \\
iCORM-2 & $55.8 \pm 9.1^{\mathrm{c}}$ & $56.2 \pm 2.1^{\mathrm{c}}$ \\
\hline
\end{tabular}

Values represent the mean \pm standard error of the mean. ${ }^{\mathrm{a}} \mathrm{P}<0.01$, vs. I/R the group; ${ }^{b} \mathrm{P}<0.01$, vs. the control group; ${ }^{\mathrm{C}} \mathrm{P}>0.05$, vs. the I/R group. CORM-2, carbon monoxide releasing molecule-2; LDH, lactate dehydrogenase; I/R, ischemia/reperfusion; MTT, methyl thiazolyltetrazolium; iCORM-2, inactive CORM-2.

diluted 1:1,000 in TBST for $1 \mathrm{~h}$ at room temperature. $\beta$-actin expression was used as the control. The intensities of the protein bands were quantified by using the image analysis software BandScan 5.0 (Glyko, Hayward, CA, USA).

Detection of cytochrome c release and $B A K / B A X$ expression. Cytosol and mitochondria fractions were analyzed by western blot analysis to quantify cytochrome $c$ and BAK/BAX release. Mitochondria were isolated as described above and lysed with lysis solution, and supernatants containing cytosol without mitochondria were also collected. Blots were probed with primary mouse anti-cytochrome $c$, BAK and BAX monoclonal antibody, respectively and then with the secondary anti-IgG antibody. $\beta$-actin expression was used as a loading control for mitochondria, as well as cytosol fractions.

Statistical analysis. SPSS 13.0 software (SPSS Inc., Chicago, IL, USA) was used for statistical analysis. Values are expressed as the mean \pm standard error of the mean for normally distributed data and median (25th, 75th percentile) for non-normally distributed data. All groups were performed in triplicate culture wells at least three times. Comparisons among the groups were performed by Kruskal-Wallis one-way analysis of variance. $\mathrm{P}<0.05$ was considered to indicate a statistically significant difference.

\section{Results}

CORM-2 protects rat neonatal cardiac myocytes against $I / R$ injury. Cells in either the control or CORM-2 groups showed an increased survival rate as compared with those subjected to $\mathrm{I} / \mathrm{R}(\mathrm{P}<0.01) . \mathrm{I} / \mathrm{R}$ induced a decrease in cell viability to $\sim 60 \%$ of control as opposed to the cells treated with CORM-2 where the cell survival was $\sim 76 \%$ of the control. There was a significant increase of LDH activity in the I/R group compared with the control group $(\mathrm{P}<0.01)$. By contrast, CORM-2 reduced the level of LDH by $\sim 45 \%(\mathrm{P}<0.01$, vs. I/R). However, the iCORM-2 group showed no changes in MTT assay and LDH level ( $\mathrm{P}>0.05$, vs. I/R) (Table I).

CORM-2 inhibits cardiomyocyte apoptosis induced by $I / R$. To evaluate the role of CORM-2 in apoptosis induced by I/R, apoptosis was assayed with a flow cytometer. Annexin V-FITC/PI 
A
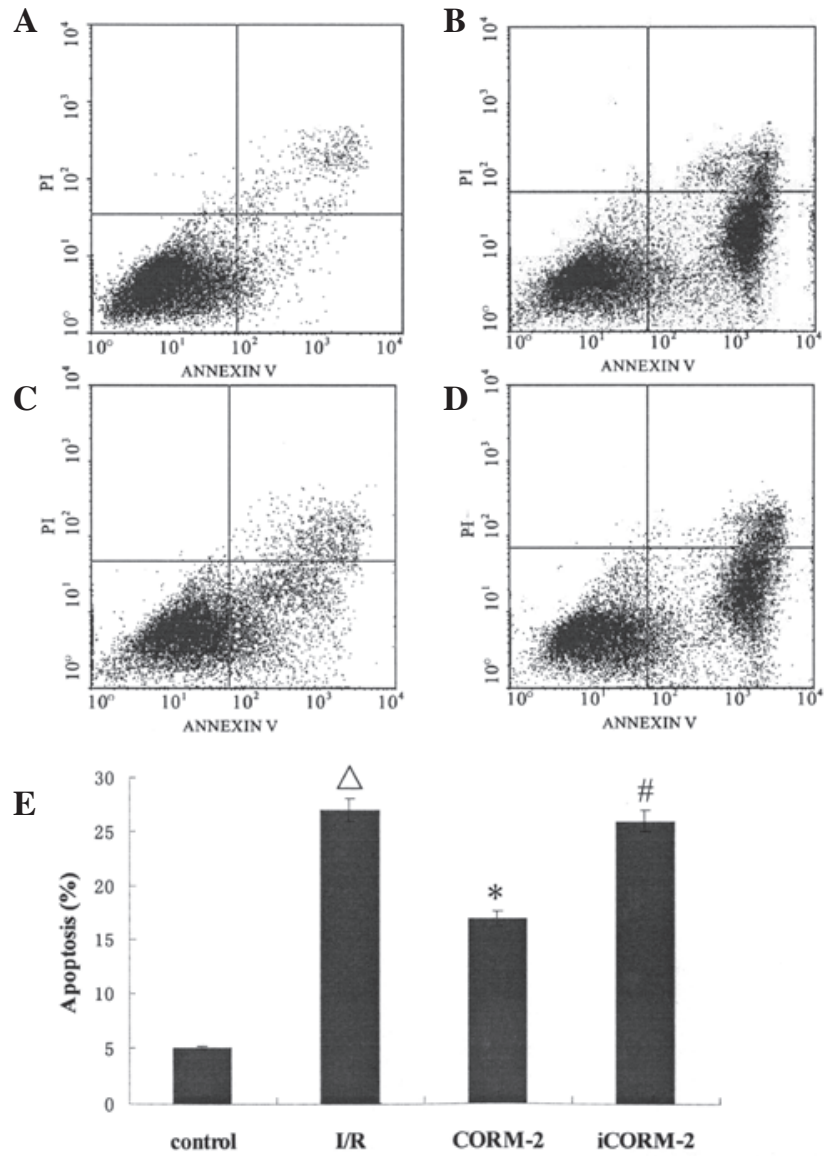

Figure 1. FITC/PI double staining and the apoptotic rate of cardiomyocytes. (A) Control group: The majority of the cells emerged at the left lower quadrant, which showed the cells were viable. (B) I/R group: The quantities of cells in the right lower quadrant increased compared with that in $(\mathrm{A})\left({ }^{\triangle} \mathrm{P}<0.01\right)$, which demonstrated that simulated I/R triggered apoptosis. (C) CORM-2 group: The quantities of cells in the right lower quadrant reduced significantly compared with that in (B) ("P $<0.01)$, suggesting that CORM-2 attenuated I/R-induced apoptosis. (D) iCORM-2 group: Cell distribution was similar to that of (B) $\left({ }^{\#} \mathrm{P}>0.05\right)$. (E) The statistical data of apoptotic rate. ${ }^{\Delta} \mathrm{P}<0.01$, vs. the control group; ${ }^{*} \mathrm{P}<0.01$, vs. the $\mathrm{I} / \mathrm{R}$ group; ${ }^{\prime \prime} \mathrm{P}>0.05$, vs. the $\mathrm{I} / \mathrm{R}$ group. FITC, Annexin V-fluorescein isothiocyanate; PI, propidium iodide; $\mathrm{I} / \mathrm{R}$, ischemia/reperfusion; CORM-2, carbon monoxide releasing molecule-2; iCORM-2, inactive CORM-2.

double staining showed that the cardiomyocytes underwent significant apoptosis when exposed to $\mathrm{I} / \mathrm{R}(\mathrm{P}<0.01$, vs. control) and CORM-2 decreased the apoptosis rate markedly $(\mathrm{P}<0.05$, vs. I/R). However, treatment with iCORM-2 did not alter the apoptotic ratio ( $\mathrm{P}>0.05$, vs. I/R) (Fig. 1).

CORM-2 attenuates ultrastructural deterioration during $I / R$. Cell apoptosis was measured by transmission electron microscopy. In the control group, there was no evident histopathological change. Margination and aggregation of nuclear chromatin, the swelling of mitochondria, and dissolution and disappearance of the mitochondrial cristae were observed in the I/R group. Similar changes were observed when the cells were combined with iCORM-2 during reperfusion. Notably, the above alterations were ameliorated in the CORM-2 group (Fig. 2).

CORM-2 improves mitochondria respiratory function. State IV and III respiration in the I/R group decreased more
A

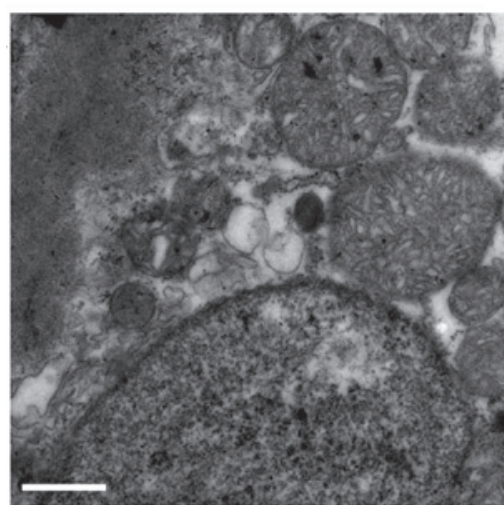

B

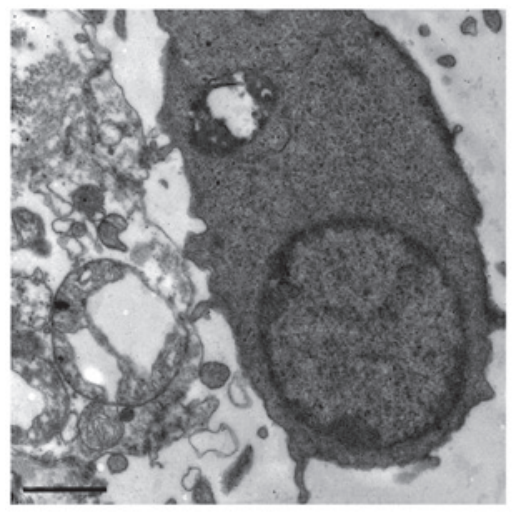

C

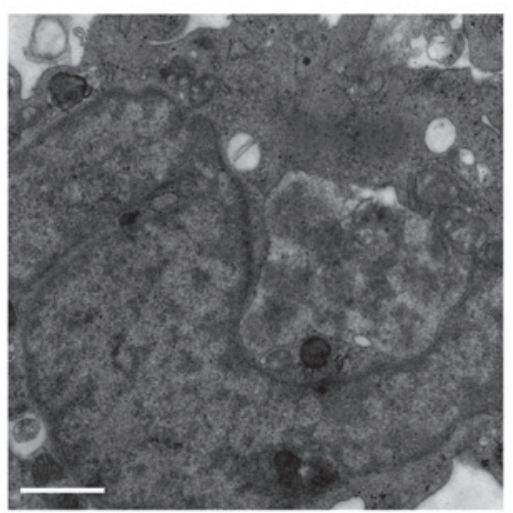

D

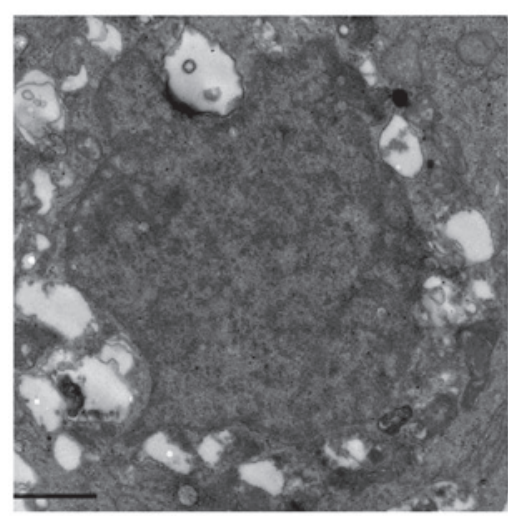

Figure 2. Ultrastructure alteration in cardiomyocytes. (A) Control, (B) I/R, (C) CORM-2, and (D) iCORM-2 groups (magnification, $\mathrm{x} 9,700$ ). There was no significant change in nuclear chromatin, mitochondria and myofilaments in the control group. In the I/R group, cardiomyocytes were observed in different degrees of cell shrinkage, nuclear chromatin margination and karyopyknosis. Mitochondrial turgidity and vacuolization were observed simultaneously. Similar changes were found in the iCORM-2 group. In the CORM-2 group, local myofilament dissolving, vacuolization of the mitochondria and disintegrated mitochondrial cristae were ameliorated to a degree, and the nuclei were almost normal. I/R, ischemia/reperfusion; CORM-2, carbon monoxide releasing molecule-2; iCORM-2, inactive CORM-2. 
Table II. Effects of CORM-2 on mitochondrial respiration in cardiomyocytes subjected to I/R injury.

\begin{tabular}{lccccc}
\hline Parameter & Control & I/R & CORM-2 & iCORM-2 & P-value \\
\hline $\begin{array}{l}\text { State IV respiration (nmol of O/min/mg } \\
\text { mitochondrial protein) }\end{array}$ & $35 \pm 2.3^{\mathrm{a}}$ & $24 \pm 3.1$ & $29 \pm 3.1^{\mathrm{a}, \mathrm{b}}$ & $23 \pm 2.9^{\mathrm{c}}$ & $<0.05$ \\
$\begin{array}{l}\text { State III respiration (nmol of O/min/mg } \\
\text { mitochondrial protein) }\end{array}$ & $221.2 \pm 11.2^{\mathrm{a}}$ & $66.7 \pm 7.7$ & $122 \pm 9.8^{\mathrm{a}, \mathrm{b}}$. & $64.4 \pm 8.3^{\mathrm{c}}$ & $<0.01$ \\
RCR & $6.32 \pm 0.7^{\mathrm{a}}$ & $2.78 \pm 1.1$ & $4.2 \pm 1.3^{\mathrm{a}, \mathrm{b}}$ & $2.8 \pm 0.9^{\mathrm{c}}$ & $<0.01$
\end{tabular}

Values are presented as the mean \pm standard error of the mean. ${ }^{\mathrm{a}} \mathrm{P}<0.01$, or $0.05 \mathrm{vs}$. I/R group; ${ }^{\mathrm{b}} \mathrm{P}<0.01$, vs. the control group; ${ }^{\mathrm{C}} \mathrm{P}>0.05$, vs the I/R group. CORM-2, carbon monoxide releasing molecule-2; I/R, ischemia/reperfusion; iCORM-2, inactive CORM-2; RCR, respiratory control ratio.
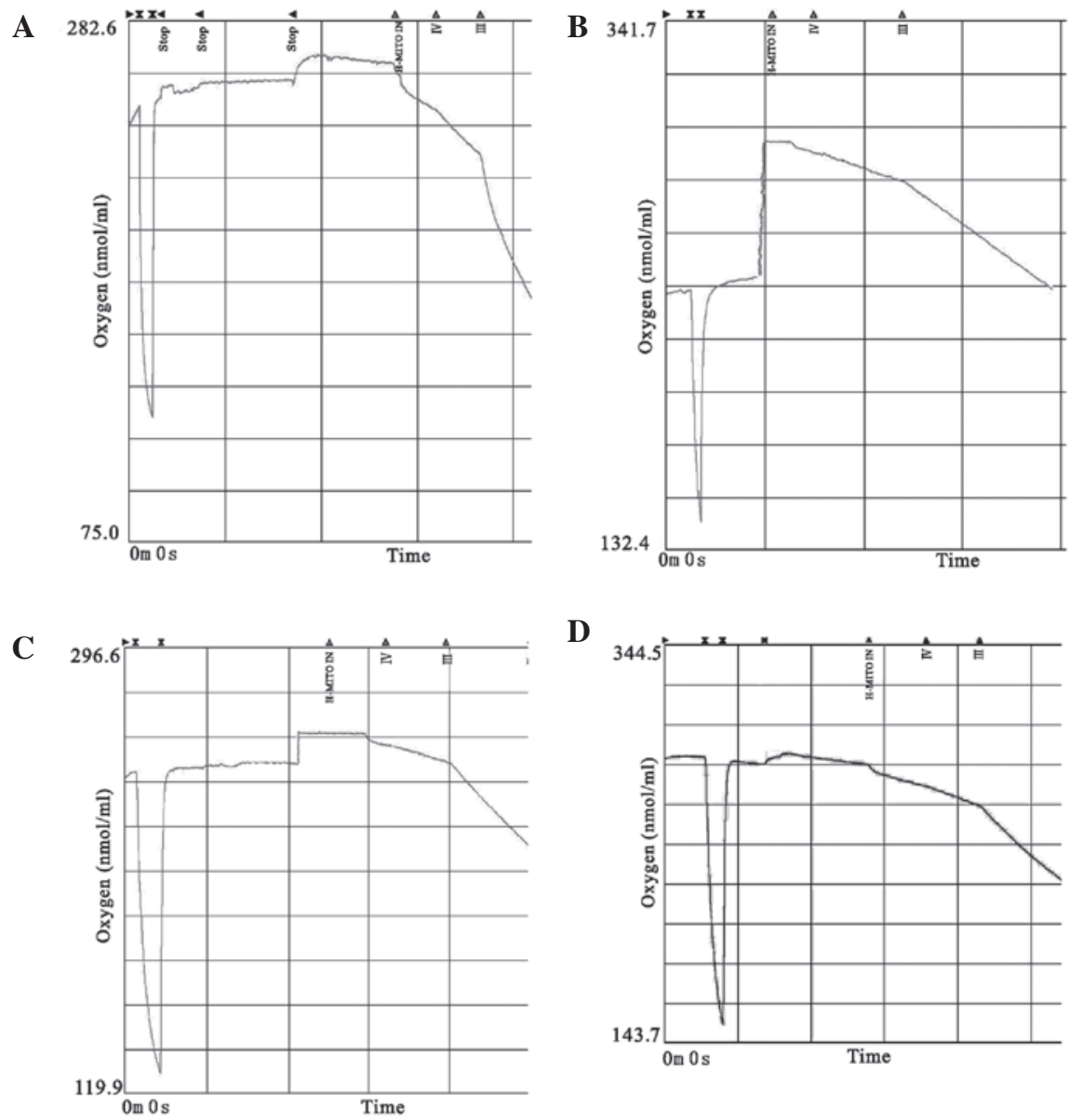

Figure 3. Representative traces of oxygen consumption of isolated mitochondria by a Clark oxygen electrode. (A) Control, (B) I/R, (C) CORM-2, and (D) iCORM-2 groups. H-MITO IN, timestamp of adding mitochondria. III, the rate of state III respiration (ADP-stimulated) in real time. IV, the rate of state IV respiration (ADP-limited) in real time. I/R, ischemia/reperfusion; CORM-2, carbon monoxide releasing molecule-2; iCORM-2, inactive CORM-2; APD, adenosine 5'-diphosphate.

significantly compared with the control group $(\mathrm{P}<0.05)$, while mitochondrial respiration was partially normalized by CORM-2 treatment $(\mathrm{P}<0.05$, vs. I/R). In comparison with the control, the I/R group showed a decreased RCR trend $(\mathrm{P}<0.05)$. CORM-2 treatment further showed a notable recovery of $\mathrm{RCR}$ despite a marked rise in state IV and III respiration $(\mathrm{P}<0.05$, vs. I/R), indicating that the latter evidently increased compared with the former. Neither the respiratory rate or $\mathrm{RCR}$ in the $\mathrm{iCORM}-2$ group showed a significant difference when compared with I/R $(\mathrm{P}>0.05)$ (Table II and Fig. 3).

Effect of CORM-2 on the expression of apoptosis-associated proteins. To further identify the pathway through which CORM-2 suppresses apoptosis, the expression of caspase-3 and the release of cytochrome $c$, as well as BAK/BAX translocation were analyzed by western blot analysis. Compared with the control group, the expression of caspase- 3 and the mito- 
A

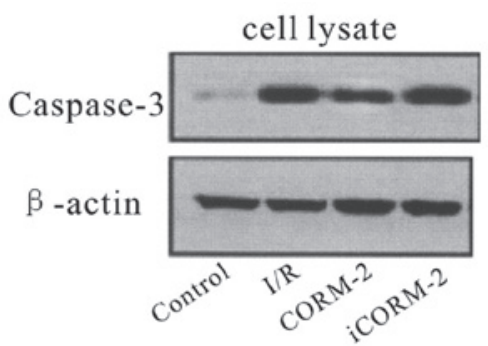

B

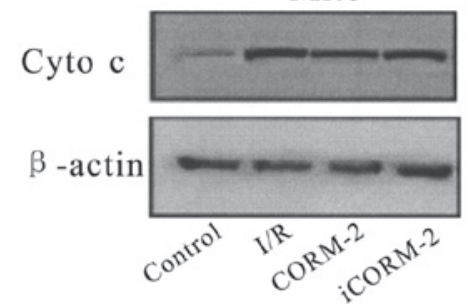

Cytosol

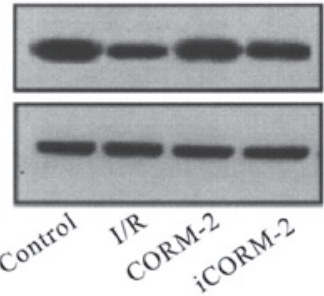

C

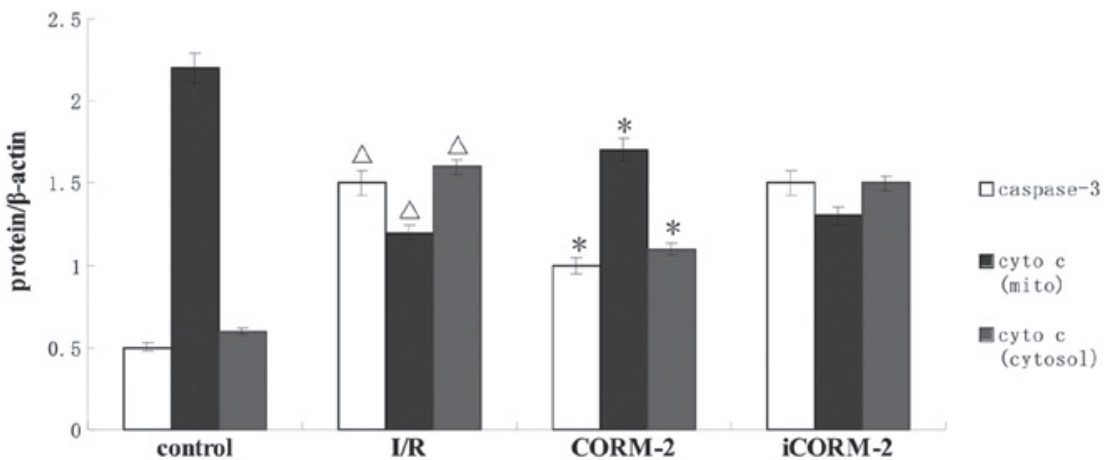

Figure 4. Detection of caspase-3 and cytochrome $c$ release in cardiomyocyte by western blot analysis. (A) The level of caspase-3 in the whole cell lysates of control, I/R, CORM-2 and iCORM-2 groups. (B) Immunoblots of rapidly separated pellets (mitochondria) and supernatants (cytosol) showed the release of cytochrome $c$ evoked by I/R. CORM-2 decreased caspased-3 expression as well as cytochrome $c$ release from mitochondria. (C) Comparative increase of caspase-3 expression and cytochrome $c$ release is shown. Cyto $c$, cytochrome $c$; mito, mitochondria; I/R, ischemia/reperfusion; CORM-2, carbon monoxide releasing molecule-2; iCORM-2, inactive CORM-2. ${ }^{*} \mathrm{P}<0.05$, vs. I/R group; ${ }^{\triangle} \mathrm{P}<0.05$, vs. control.

A

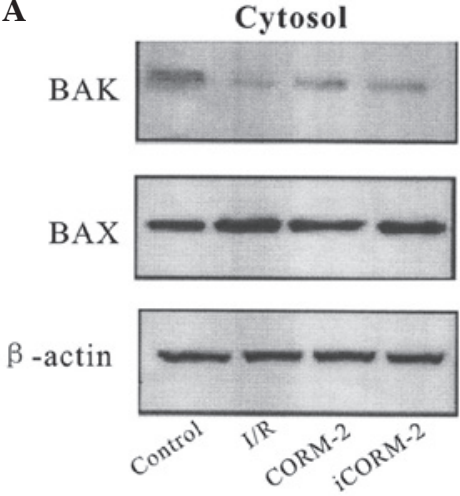

B

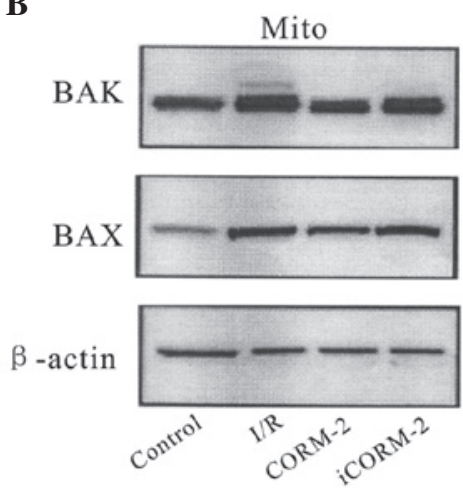

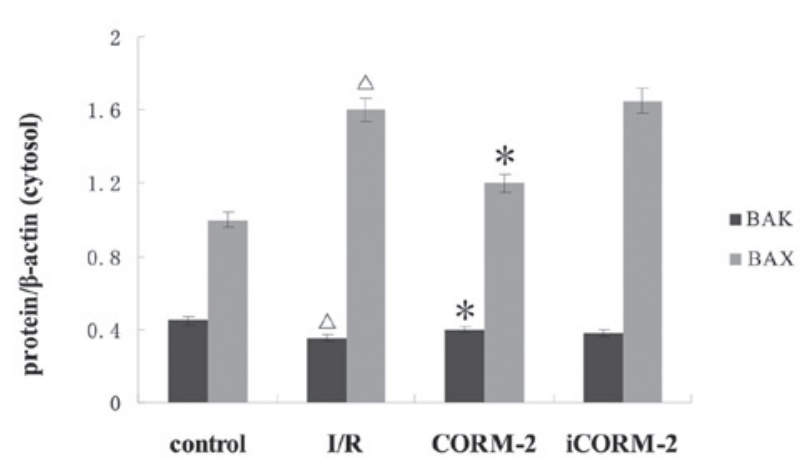

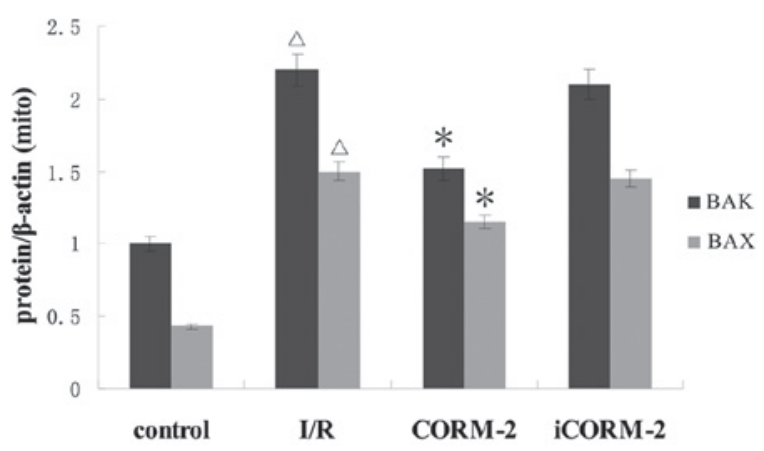

Figure 5. Detection of BAK/BAX expression in cardiomyocytes by western blot analysis. (A) Immunoblots showed the presence of BAX and very small amounts of BAK in the cytosol of control, I/R, CORM-2 and iCORM-2 groups, respectively. (B) Immunoblots of rapidly separated mitochondria showed that a boost in BAK/BAX expression suffered from I/R could be reversed by CORM-2, but not by iCORM-2. Cumulative data of BAK/BAX relative expression of the cytosol and mitochondria fractions is shown in the lower panel, respectively. ${ }^{*} \mathrm{P}<0.05$, vs. I/R group; ${ }^{\triangle} \mathrm{P}<0.05$, vs. control. I/R, ischemia/reperfusion; CORM-2, carbon monoxide releasing molecule-2; iCORM-2, inactive CORM-2. 
chondrial release of cytochrome $c$ were markedly increased in the $\mathrm{I} / \mathrm{R}$ group $(\mathrm{P}<0.05)$ and CORM-2 treatment reversed these increases $(\mathrm{P}<0.05$, vs. I/R). The cytosol maintained some low-level BAK content in all groups $(\mathrm{P}>0.05)$, while mitochondria contained medium-level BAK in the control group. The western blot assay further showed a sharp increase of BAK content evoked by $\mathrm{I} / \mathrm{R}$ in the mitochondria $(\mathrm{P}<0.05$, vs. control), where CORM-2 treatment partially restored the level of BAK $(\mathrm{P}<0.05$, vs. I/R). Immunoblots in the control group showed the majority of the BAX in the cytosol, whereas isolated mitochondria showed weakly positive expression. By contrast, $\mathrm{I} / \mathrm{R}$ increased the BAX expression level in the mitochondria and induced a continuous increase in the cytosol $(\mathrm{P}<0.05$, vs. control). Notably, BAX expression evoked by I/R was partially inhibited by CORM-2 treatment $(\mathrm{P}<0.05)$. iCORM-2 did not have any effect ( $\mathrm{P}>0.05$, vs. I/R) (Figs. 4 and 5).

\section{Discussion}

The results of five independent assays, biochemical indexes, Annexin V-FITC/PI double staining using flow-cytometery, measurement of mitochondrial respiratory rates, the ultrastructure changes observed by transmission electron microscopy, protein detection, including BAK/BAX, as well as cytochrome $c$ and the late apoptotic protease, caspase-3, showed that exogenous CO released from CORM-2 alleviated I/R-induced apoptosis of primary cultured myocardial cells from newborn SD rats. The study further supported the hypothesis that CORM-2 may attenuate myocardial apoptosis by inhibiting BAK/BAX-mediated intrinsic pathway. Since iCORM-2, which is chemically identical to CORM-2 but does not liberate $\mathrm{CO}$, had no effect, the attenuated injury observed in the CORM-2-treated group must be ascribed to the effect of CO. In addition, CORM-2 treatment began at the time of reperfusion, therefore the experimental instructions employed in the present study have clinical relevance for the treatment of patients with acute myocardial infarction and cardiac arrest.

Cardiomyocyte apoptosis is a major pathogenesis during myocardial I/R injury. In the present study, the I/R-induced cell viability and LDH activity were evaluated, which are indicators of myocardial membrane injury. Following $2 \mathrm{~h}$ ischemia, followed by $4 \mathrm{~h}$ reperfusion, the cell viability was markedly reduced with LDH levels in the culture medium, 2-fold higher compared with the control group. These changes were comparable with flow cytometry data, which indicated myocardial apoptosis. Furthermore, the marked ultrastructural alterations, as well as dysfunction of mitochondrial respiration triggered by $\mathrm{I} / \mathrm{R}$, indicated that disrupted energy metabolism and dysregulation of cell death may target mitochondria. However, CORM-2 treatment significantly prevented I/R-induced myocardial injury confirmed by increased viability, reduced apoptosis rate, as well as an improvement of morphology and respiratory function, suggesting that CORM-2 may play a positive role in cardioprotection through the promotion of metabolism and inhibition of mitochondrion-mediated apoptosis.

To investigate this hypothesis, the expression of pro-apoptotic molecules BAK and BAX in the mitochondria and cytoplasm without the mitochondrial part, respectively, was examined. By contrast with BAX, which is concentrated in the cytosol in an inactive state and targets to the MOM upon apoptotic stimuli, monomeric inactive conformer of BAK is detected to interact specifically with a voltage-dependent anion channel (VDAC) isoform, $\mathrm{VDAC}_{2}$, within MOM by protein cross-linking (19). Conditional deletion of BAK abrogated the increased ionomycin-induced apoptosis of $\mathrm{VDAC}_{2}$-deficint thymocytes (20). A number of apoptotic agents activate several BAK/BAX-dependent mechanisms that execute the permeabilization of mitochondrial membranes (21), which are required for cell death by mitochondrial or intrinsic pathways. Upon apoptosis, the 'activator' BH3-only members, including tBID, $\mathrm{BAD}, \mathrm{BIM}$ and PUMA, trigger BAK and BAX to convert from the monomeric form to homo-oligomers, which mediate the efflux of cytochrome $c$ from mitochondria and leads to the activation of caspases (22-24). In the present study, western blot analysis of the cytosolic and mitochondrial fraction separated at the end of RCR measurement indicated an increase in BAX expression in the cytosol and translocation of BAX from the cytosol to the mitochondria following reperfusion. By contrast, BAK did not alter in the cytosol, but increased in the mitochondria. The enhanced expression of BAK/BAX in mitochondria as well increased cytochrome $c$ in the cytosol suggested that BAK/BAX may be involved in the rupture of the MOM and may mediate intrinsic apoptosis in triggering I/R. Wei et al (21) observed activation and oligomerization of BAK/BAX following tBID treatment, whereas $\mathrm{BAK}^{-/-}$and $\mathrm{BAX}^{-/-}$MEFs were resistant to apoptotic death by mitochondria-dependent intrinsic signals. At present the mechanisms by which BAK and BAX cause the MOM to become permeable are not well understood. A number of studies suggested that $\mathrm{BAK}$ and $\mathrm{BAX}$ regulate mitochondrial morphology machinery to activate MOM permeabilization (25), form large pores themselves (26), or change the conformation of existing pores (27) and may attribute in part to cytochrome $c$ release and caspase activation.

Another important finding is that the mitochondrial respiratory response to simulated ischemia and reperfusion is involved in decreased respiratory rates of state III (ADP-stimulated respiration) and IV (non-ADP-stimulated respiration), as well as depressing RCR, indicating a mitochondrial proton leak and uncoupling of oxidative phosphorylation. These findings are consistent with a previous study which demonstrated that $\mathrm{I} / \mathrm{R}$ caused a decrease in liver mitochondrial state III respiration rate, as well as RCR and an increase in state IV respiration rate in the presence of exogenous cytochrome $c$, suggesting the disruption of MOM (28). Previous studies have reported that a reduction in RCR may be due to no change, or an increase in state IV respiration, and a decrease in state III respiration $(29,30)$. The variance of these results may be due to the duration of ischemia and the degree of reperfusion injury, as well as the difference between in vitro and in vivo experiments. It has been also largely accepted that mitochondrial uncoupling leads to a reduction in mitochondrial ATP synthesis and $\mathrm{NADP}(\mathrm{H})$ content, which were all associated with membrane damage (31). Furthermore, mitochondrial swelling with membrane permeabilization observed may be partially responsible for injured mitochondrial respiratory function and decreased mitochondrial membrane potential.

Increasing evidence has shown the cardioprotective action of CORMs with transition metal carbonyls as carriers, 
and corroborated the hypothesis that $\mathrm{CO}$ is a homeostatic and cytoprotective gaseous mediator required as an adjuvant to ameliorate apoptotic processes, as well as inflammation and oxidative stress (32). The mechanisms underlying $\mathrm{CO}$-induced cardioprotection remain to be elucidated, despite $\mathrm{CO}$ holding beneficial bioactive properties on a number of tissues. Recent investigations have implicated the mitochondria as a prime target for cytoprotection and therapy of $\mathrm{CO}$ (11). The present results indicated that CORM-2 may inhibit simulated I/R-induced apoptosis via a $\mathrm{BAK} / \mathrm{BAX}$-mediated intrinsic pathway. $\mathrm{CO}$ has been found to promote the anti-apoptotic protein BCL-2 expression in lung ischemia models (33). However, it was worth noting in the study that the anti-apoptosis effect of $\mathrm{CO}$ may be partly attributed to the inhibition of pro-apoptotic BAK/BAX protein expression. Another direct method for assessing the effect of CORM-2 on mitochondria is cellular oxygen consumption. CORM-2, at a low concentration, was observed to increase respiratory rates of state III and IV, as well as RCR with an $20 \%$ reduction from normal level. CO released by CORM-2 is hypothesized to partially reduce BAK/BAX content in the mitochondria, which contributes to maintaining MOM integrity and transmembrane potential, as well as the tightness of coupling between respiration and phosphorylation. This is in agreement with a previous study where treatment with CORMs during kidney cold storage increased mitochondrial respiratory control index at reperfusion (34). However, the results of associated studies on mitochondrial respiration in response to $\mathrm{CO}$ have been inconsistent. Astrocytes isolated from the cortex presented an increase of oxygen consumption at $36 \mathrm{~h}$ following one brief exposure to CO (12). Different experimental conditions, including early or late response and concentration or period of exposure may alter the mitochondrial coupling state and oxidative metabolism. Other investigators have claimed that $\mathrm{CO}$ biological function was associated with mitochondrial mild uncoupling state, which decreased cell respiration, as well as excessive and toxic mitochondrial reactive oxygen species production. Inhibition of uncoupling proteins (UCP) or blockade of adenine nucleotide transporter (ANT) attenuated the effect, indicating that CO may open UCP and/or ANT at a mild uncoupling state (35).

It should be noted that the current study may have a number of limitations. Firstly, CORM-2 did not fully recover the mitochondrial respiration in the study, thus further experiments are required to observe whether it maintained the electron transport chain in a mild uncoupling state or not. Secondly, it may not directly prove the association between impaired mitochondrial respiration and BAK/BAX activation, thus, more convincing evidence is required to support the hypothesis. Finally, further dose-dependent anti-apoptotic properties of CORM-2, as well as its side effects should be evaluated in in vitro or in vivo experiments, although such a dose of CORM-2 appeared to be effective and safe in this study.

In conclusion, the present study suggested that exogenous CO released from CORM-2 may protect cardiomyocytes from I/R-induced apoptosis. It may be associated with the inhibition of a BAK/BAX-dependent mitochondrial pathway and the improvement of energy metabolism.

\section{Acknowledgements}

The authors would like to thank Jinlang Wu (Department of Electron Microscopy, Sun Yat-sen University) for his valuable assistance with the experimental technique and image analysis.

\section{References}

1. Bialik S, Cryns VL, Drincic A, Miyata S, Wollowick AL, Srinivasan A and Kitsis RN: The mitochondrial apoptotic pathway is activated by serum and glucose deprivation in cardiac myocytes. Circ Res 85: 403-414, 1999.

2. Radhakrishnan J, Wang S, Ayoub IM, Kolarova JD, Levine RF and Gazmuri RJ: Circulating levels of cytochrome $c$ after resuscitation from cardiac arrest: a marker of mitochondrial injury and predictor of survival. Am J Physiol Heart Circ Physiol 292: H767-H775, 2007.

3. Li H, Fang X, Yang Z, et al: Ischemia hypothermia improved contractility under normothermia reperfusion in the model of cultured cardiomyocyte. In Vitro Cell Dev Biol Anim 48: 284-292, 2012.

4. Di Lisa F, Canton M, Menabò R, Kaludercic N and Bernardi P: Mitochondria and cardioprotection. Heart Fail Rev 12: 249-260, 2007.

5. Danial NN and Korsmeyer SJ: Cell death: critical control points. Cell 116: 205-219, 2004

6. Chipuk JE and Green DR: How do BCL-2 proteins induce mitochondrial outer membrane permeabilization? Trends Cell Biol 18: 157-164, 2008.

7. Kubli DA, Ycaza JE and Gustafsson AB: Bnip3 mediates mitochondrial dysfunction and cell death through Bax and Bak. Biochem J 405: 407-415, 2007.

8. Ren D, Tu HC, Kim H, et al: BID, BIM, and PUMA are essential for activation of the BAX- and BAK-dependent cell death program. Science 330: 1390-1393, 2010.

9. Roy SS, Ehrlich AM, Craigen WJ and Hajnóczky G: VDAC2 is required for truncated BID-induced mitochondrial apoptosis by recruiting BAK to the mitochondria. EMBO Rep 10: 1341-1347, 2009.

10. Motterlini R and Otterbein LE: The therapeutic potential of carbon monoxide. Nat Rev Drug Discov 9: 728-743, 2010.

11. Queiroga CS, Almeida AS and Vieira HL: Carbon monoxide targeting mitochondria. Biochem Res Int 2012: 749845, 2012.

12. Almeida AS, Queiroga CS, Sousa MF, Alves PM and Vieira HL: Carbon monoxide modulates apoptosis by reinforcing oxidative metabolism in astrocytes: role of Bcl-2. J Biol Chem 287: 10761-10770, 2012.

13. Conde de la Rosa L, Vrenken TE, Hannivoort RA, et al: Carbon monoxide blocks oxidative stress-induced hepatocyte apoptosis via inhibition of the p54 JNK isoform. Free Radic Biol Med 44: 1323-1333, 2008.

14. Bannenberg GL and Vieira HL: Therapeutic applications of the gaseous mediators carbon monoxide and hydrogen sulfide. Expert Opin Ther Pat 19: 663-682, 2009.

15. Motterlini R: Carbon monoxide-releasing molecules (CO-RMs): vasodilatory, anti-ischaemic and anti-inflammatory activities. Biochem Soc Trans 35: 1142-1146, 2007.

16. Katada K, Bihari A, Mizuguchi S, et al: Carbon monoxide liberated from CO-releasing molecule (CORM-2) attenuates ischemia/reperfusion (I/R)-induced inflammation in the small intestine. Inflammation 33: 92-100, 2010.

17. Caumartin Y, Stephen J, Deng JP, et al: Carbon monoxide-releasing molecules protect against ischemia-reperfusion injury during kidney transplantation. Kidney Int 79: 1080-1089, 2011.

18. Gomez LA, Alekseev AE, Aleksandrova LA, Brady PA and Terzic A: Use of the MTT assay in adult ventricular cardiomyocytes to assess viability: effects of adenosine and potassium on cellular survival. J Mol Cell Cardiol 29: 1255-1266, 1997.

19. Cheng EH, Sheiko TV, Fisher JK, Craigen WJ and Korsmeyer SJ: VDAC2 inhibits BAK activation and mitochondrial apoptosis. Science 301: 513-517, 2003.

20. Ren D, Kim H, Tu HC, et al: The VDAC2-BAK rheostat controls thymocyte survival. Sci Signal 2: ra48, 2009.

21. Wei MC, Zong WX, Cheng EH, et al: Proapoptotic BAX and BAK: a requisite gateway to mitochondrial dysfunction and death. Science 292: 727-730, 2001. 
22. Wei MC,Lindsten T,Mootha VK, et al: tBID, a membrane-targeted death ligand, oligomerizes BAK to release cytochrome $c$. Genes Dev 14: 2060-2071, 2000.

23. Cheng EH, Wei MC, Weiler S, Flavell RA, Mak TW, Lindsten T and Korsmeyer SJ: BCL-2, BCL-X(L) sequester BH3 domain-only molecules preventing BAX- and BAK-mediated mitochondrial apoptosis. Mol Cell 8: 705-711, 2001.

24. Kim H, Rafiuddin-Shah M, Tu HC, Jeffers JR, Zambetti GP, Hsieh JJ and Cheng EH: Hierarchical regulation of mitochondrion-dependent apoptosis by BCL-2 subfamilies. Nat Cell Biol 8: 1348-1358, 2006.

25. Karbowski M, Norris KL, Cleland MM, Jeong SY and Youle RJ: Role of Bax and Bak in mitochondrial morphogenesis. Nature 443: 658-662, 2006.

26. Youle RJ and Strasser A: The BCL-2 protein family: opposing activities that mediate cell death. Nat Rev Mol Cell Biol 9: 47-59, 2008.

27. Shoshan-Barmatz V, Keinan $\mathrm{N}$ and Zaid $\mathrm{H}$ : Uncovering the role of VDAC in the regulation of cell life and death. J Bioenerg Biomembr 40: 183-191, 2008.

28. Trumbeckaite S, Kincius M, Preidis A, Preidiene M, Veikutis V, Borutaite V and Gulbinas A: Effects of ischemia-reperfusion and pretreatment with mildronate on rat liver mitochondrial function. Pharmacol Rep 61: 859-869, 2009.

29. Venditti P, Masullo P and Di Meo S: Effects of myocardial ischemia and reperfusion on mitochondrial function and susceptibility to oxidative stress. Cell Mol Life Sci 58: 1528-1537, 2001.
30. Chen Q, Moghaddas S, Hoppel CL and Lesnefsky EJ: Reversible blockade of electron transport during ischemia protects mitochondria and decreases myocardial injury following reperfusion. J Pharmacol Exp Ther 319: 1405-1412, 2006.

31. Elimadi A, Settaf A, Morin D, Sapena R, Lamchouri F, Cherrah Y and Tillement JP: Trimetazidine counteracts the hepatic injury associated with ischemia-reperfusion by preserving mitochondrial function. J Pharmacol Exp Ther 286: 23-28, 1998 .

32. Clark JE, Naughton P, Shurey S, et al: Cardioprotective actions by a water-soluble carbon monoxide-releasing molecule. Circ Res 93: e2-e8, 2003.

33. Zhang X, Shan P, Alam J, Davis RJ, Flavell RA and Lee PJ: Carbon monoxide modulates Fas/Fas ligand, caspases, and Bcl-2 family proteins via the $\mathrm{p} 38$ alpha mitogen-activated protein kinase pathway during ischemia-reperfusion lung injury. J Biol Chem 278: 22061-22070, 2003.

34. Sandouka A, Fuller BJ, Mann BE, Green CJ, Foresti R and Motterlini R: Treatment with CO-RMs during cold storage improves renal function at reperfusion. Kidney Int 69: 239-247, 2006.

35. Lo Iacono L, Boczkowski J, Zini R, Salouage I, Berdeaux A, Motterlini R and Morin D: A carbon monoxide-releasing molecule (CORM-3) uncouples mitochondrial respiration and modulates the production of reactive oxygen species. Free Radic Biol Med 50: 1556-1564, 2011. 